\title{
Efficient strategies for selecting small targets on pen- based systems: an evaluation experiment for selection strategies and strategy classifications
}

\author{
Xiangshi Ren and Shinji Moriya \\ Department of Information and Communication Engineering, Tokyo Denki University, 2-2 \\ Kanda-Nishikicho, Chiyoda-ku, Tokyo 101-8457, Japan \\ Tel: +81-3-5280-3335, Fax: +81-3-5280-3564 \\ ren@c.dendai.ac.jp,moriya@c.dendai.ac.jp
}

\begin{abstract}
This paper describes six strategies for selecting small targets on pen-based systems. We have classified the strategies into strategy groups according to their characteristics. An experiment was conducted comparing selection time, error rate and user preference ratings for the six selection strategies. We focused our attention on the three variables associated with pen-based selection: size, direction and distance to target. Three target sizes, eight penmovement-directions and three pen-movement-distances were applied to all six strategies. Experimental results show that the best strategy was the "Landon2" strategy when the strategies were evaluated individually, and the best strategy group was the "In-Out" strategy group when evaluated in groups. Analyses also showed that differences between strategies were influenced by variations in target size, however, they were not influenced by pen-movementdistance and pen-movement-direction. Analyses of grouped strategies produced the same results. Ideas for future research are also presented.
\end{abstract}

Key words: mobile computing, pen-based input interfaces, selection strategies, classifications of selection strategies, small targets, differences between selection strategies.

\section{INTRODUCTION}

The advent of mobile computing has meant that pen-based computers have created a large niche in the computer market. Pen-based input is well suited to jotting down text and accessing information in mobile computing situations.

The original version of this chapter was revised: The copyright line was incorrect. This has been corrected. The Erratum to this chapter is available at DOI: 10.1007/978-0-387-35349-4_22 
However, not enough empirical tests have been performed to measure how we can improve its usage.

We pay attention to the problems as follows: (1) In small pen-based systems, attempts are more often made to access information than to input information. Access includes the selection of menus, data (one character of the text or graphic segment, etc.), ranges etc., or the selection of a software keyboard displayed on a screen (Soukoreff and Mackenzie 1995). Data input includes handwriting input for both recognition and non-recognition applications. (2) As the amount of information displayed on the screen increases, users have to select smaller targets. This tendency is especially obvious in mobile products, such as personal digital assistants (PDAs), personal information managers (PIMs), and other mobile penbased applications. The trade-off between the accessibility of targets and the amount of information presented is a fundamental problem in human-computer design. In order to solve the problem, some leading studies have developed a variety of relatively efficient selection strategies for the touchscreen (Potter, Weldon, and Shneiderman, 1988; Sear, Plaisant, and Shneiderman, 1992), the mouse (Brewster, et al., 1995; Kabbash and Buxton, 1995; Worden et al., 1997), and 3D input systems (Zhai, Buxton, and Milgram, 1994).

However, current target selection strategies for pen-based systems are mostly only imitations of selection techniques for mouse and touch-screen devices. Our previous papers have addressed the problem and described an experiment which compared strategies on a pen-based system (e.g. Ren and Moriya, 1997a). However, studies that identify and quantify the influential factors that make strategies more or less efficient remained a challenge. This paper addresses this issue. The work reported here is part of a project that looks at selection strategy problems in interface design (Ren, 1996; Ren and Moriya, 1997a, 1997b, 1998).

In section 2, we describe the six strategies and their classification. In section 3 , we describe the experiment, the procedure, the design, and the aims. In section 4 , we show the results of the analyses. In section 5 , we identify the best strategies from the six individual strategies and from the strategy groups. We also evaluate the influence of the variables on the differences between the strategies and the strategy groups. In section 6 , we present ideas for future research.

\section{THE SIX STRATEGIES AND THEIR CLASSIFICATION}

\subsection{Tablet structure and the six strategies}

An electromagnetic tablet was used in the experiment. When the pen-tip is within a given height above the tablet surface $(1 \mathrm{~cm})$, the computer can recognise the co-ordinates $(x, y)$ of the pen-tip. Thus, even though the menu on the screen is 2 dimensional (2D), it can be highlighted or selected when the pen is above the tablet surface (within $1 \mathrm{~cm}$ ). This means that the menu can be expressed as a 3 dimensional (3D) target. 


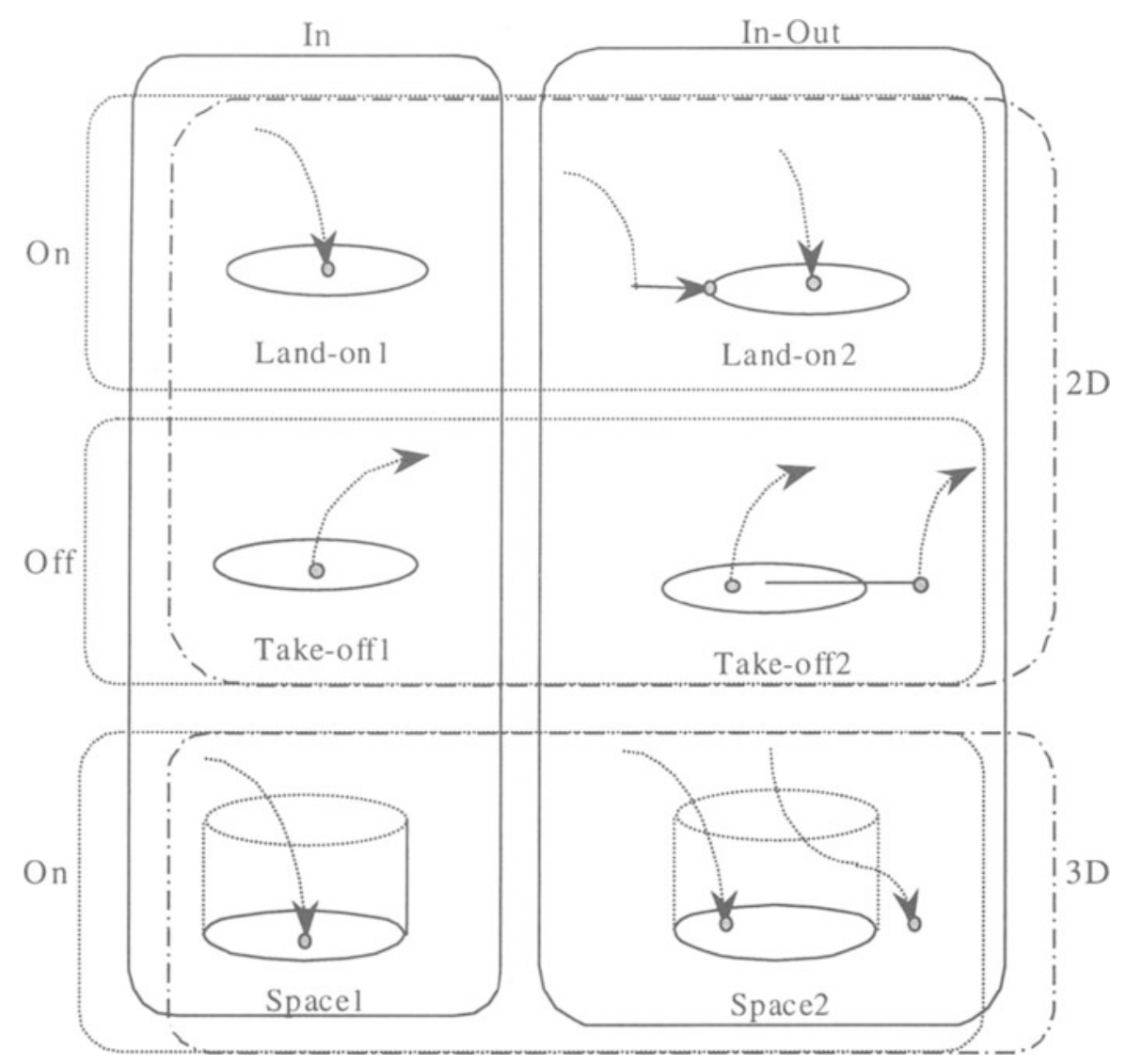

Figure 1 Classification of the six strategies.

The six strategies are illustrated in Figure 1. The ellipse and the cylinder shown in Figure 1 illustrate targets on the screen. The ellipse represents a $2 \mathrm{D}$ circular target. The cylinder represents a $3 \mathrm{D}$ target. That is, the circle with a solid line is at the bottom of the 3D target. Some responses will take place when the pen is in the cylinder. It is important to note that although the illustration in Figure 1 shows circular targets, the shape of the target has no definitive bearing on this discussion. The arrow shows the movement of the pen-tip. A dashed line arrow means the pentip is above the screen and a solid line arrow shows that the pen-tip is on the screen. The point (small dark circle) shows where the target selection is made by the pen.

The six strategies for selecting a target in the experiment are as follows:

- Land-on1 strategy: the pen approaches from above. The target is selected only momentarily at the time the pen makes contact with the screen in the target area.

- Land-on2 strategy is an extension of the Land-on1 strategy. Here also the target is selected when the pen touches it for the first time, but in this case the pen lands outside the target area before moving into it.

- Take-off1 strategy: the target is highlighted only while the pen is touching it. The selection is made at the moment the pen is taken off the target. 
- Take-off2 strategy is an extension of the Take-off1 strategy. The target is highlighted only while the pen is in contact with it, however the selection is made when the pen is removed from any point on the screen either inside or outside the target area.

- Spacel strategy: the pen approaches from above. The target is highlighted while the pen is within the $1 \mathrm{~cm}$ high cylinder above the target. Selection is made at the moment the pen makes contact with the target area (i.e. inside the bottom circle).

- Space2 strategy is an extension of the Spacel strategy. The target is highlighted while the pen is within the $1 \mathrm{~cm}$ high cylinder above the target. After highlighting, the selection is made when the pen makes contact with any point on the screen either inside or outside the target area.

The Land-on 1 and Take-off1 strategies are already in common use. The Landon2 strategy corresponds to the first-contact strategy (Potter, Weldon, and Shneiderman, 1988). The Take-off2, Spacel and Space2 strategies were new strategies designed by Ren and Moriya (1997a).

\subsection{Characteristics of the Six Strategies and their Classification}

What characteristics do these various strategies have? What criteria were used to select the six strategies? Regarding the above questions, we concentrated on the six conditions created by the pen parameters (Ren and Moriya, 1995). They are: contact with the screen, removal from the screen, contact inside the target, contact outside the target, target highlighted and target not highlighted.

Figure 1 shows classification of the six strategies according to their characteristics.

- 2D and 3D strategies: Targets exist both as planes (2D) and as solid bodies (3D). Here, the 2D strategies are the Land-on1, Land-on2, Take-off1 and Takeoff 2 strategies. The 3D strategies are the Space1 and Space2 strategies.

- On and Off strategies: Contact and removal of the pen were considered as movements between the $2 \mathrm{D}$ plane and $3 \mathrm{D}$ space. Pen contact involves a movement from $3 \mathrm{D}$ to $2 \mathrm{D}$, while removal involves a movement from $2 \mathrm{D}$ to $3 \mathrm{D}$. These interactions were considered to be suitable conditions for the subject to recognise and confirm the moment of target selection. The strategies in which selection was made by contact with the screen (Land-on1, Land-on2, Space1 and Space2 strategies) were named On strategies. The strategies in which selection was made by removal from the screen (Take-off1 and Take-off 2 strategies) were named Off strategies. Where the target existed on the 2D plane, both the On and Off strategies were deployed. Where the target existed in $3 \mathrm{D}$ space, only the On strategies were used, assuming that the pen was approaching the target from above.

- In and In-Out strategies: We considered the movement of the pen into and out of the target from the perspective of the user's eyes and ears. When the pen moved into or out of the target, users could confirm whether or not the target was highlighted. Those strategies in which selection was made by contact 
within the target area were named In strategies (the Land-on 1, Take-off 1 and Space 1 strategies). On the other hand, those strategies in which selection was made by contact or removal either inside or outside the target were named InOut strategies (Land-on2, Take-off2 and Space2 strategies).

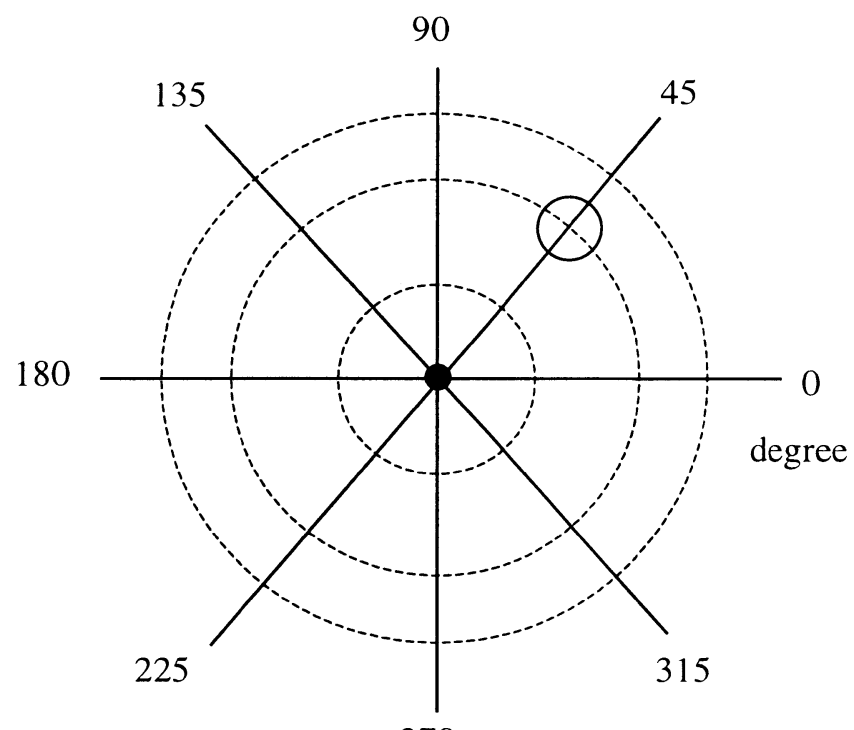

270

Figure 2 An example of the display of a target. The black dot is the initial position. The small circle shows one of the twenty-four possible positions for the display of a target. The dotted line shows the pen-movement-distances from the initial position to the target. The solid line indicates the pen-movement directions to the target from the initial position.

\section{THE EXPERIMENT}

\subsection{Subjects}

Twenty-one subjects (17 male, 4 female; all right-handed, university students), were tested for the experiment. Their ages ranged from twenty-one to twenty-three years. Ten had had previous experience with pen-input systems, while the others had had no experience. 


\subsection{Apparatus}

The hardware used in this experiment was: an electromagnetic tablet-cumdisplay (HD-640A, WACOM Corp.), a stylus pen (SP-200A, WACOM Corp.), and a personal computer (PC9801-DA, NEC Corp.). The space resolution of the tablet input was $0.05 \mathrm{~mm}$ per point. The height of the liquid crystal screen was $144.0 \mathrm{~mm}$ and the width was $230.4 \mathrm{~mm}$. The liquid crystal display resolution was $600 \times 400$ pixels. 1 pixel was about $0.36 \mathrm{~mm}$. The pen/screen contact area was $1.40 \mathrm{~mm}$ in diameter.

\subsection{Procedure}

First the experiment was explained to each subject. Each of them had 20 practice trials immediately before the experiment started. The message "Select a target as quickly and accurately as possible using the strategy" was displayed on the screen of the experimental tool when the experiment started.

When a target was being selected using any one of the strategies, the steps were as follows (Figure 2): (a) initial position: a circular initial position was displayed at the centre of the screen. The initial position was the place where the pen was pointed immediately before beginning the selection procedure. The subject had been told which strategy he/she was to use and how many trails he/she had to do. (b) Touching at the initial position: the subject touched the initial position with the pen. (c) Display of a target: the target was displayed with size and position changed at random by the computer. Targets of a particular size were never displayed in the same position twice. The distances between the initial position and the target were 39,131 or 160 pixels, randomly selected by the computer. (d) Target selection: the subject received a message on the screen to indicate whether he/she had made a successful selection or not. (e) The subject then repeated (a) and (d) above. (f) End of test: a message indicating the end of the test was displayed when the subject had completed the task.

After they finished testing each strategy, the subjects were asked to fill in a questionnaire. The first question was: "For the strategy tested just now, when selecting T, how do you rate P? Please answer on a 1-to-5 scale (1 2334 5)". Here, $1=$ lowest preference, and $5=$ highest preference. " $\mathrm{T} "$ means large or small targets as tested in the particular trial. "P" consisted of the six sub-questions regarding selection accuracy, selection speed, selection ease, learning ease, satisfaction and desire to use The questions (P) were asked of both large and small target sizes in each strategy. The second question was: "Which positions (i.e. direction and distances) were most comfortable for selecting the targets in the strategy? ". The subject marked his/her preferences on Figure 2.

The strategies were not mixed. In a given trial each subject used only one strategy. The data for each strategy were recorded automatically as follows:

(1) Presence or absence of error when a target was selected. One selection was a continuous operation from the moment the pen touched the initial position until the removal of the pen from the tablet surface. Feedback to the subject indicated 
whether the selection was successful or not. In either case, the subject could not cancel the selection.

(2) Position and size of the target displayed.

(3) The time lapsed between display of the target and the moment when the pen contacted the screen.

(4) The time lapsed between contact with the target and removal from the screen.

(5) The time lapsed between contact with the screen and contact with the target.

These times were measured to an accuracy of $10 \mathrm{~ms}$ using a special program.

Data as defined in item (3) was recorded for the Land-on1, Space1 and Space2 strategies. Data as defined in item (5) above was recorded for the Land-on2 strategy. Data as defined in item (4) above was recorded for the Take-offl and Take-off 2 strategies.

\subsection{Design}

The experiment used a mixed factorial design. (1) Size of target: To examine the relationship between target size and strategy, three target sizes of 3,5 and 9 pixels $(1.1 \mathrm{~mm}, 1.8 \mathrm{~mm}$, and $3.2 \mathrm{~mm}$ diameter circles) were used in all trials. All the targets for the experiment were circular. Circular targets were used so that the distance between the initial position and the edge of all targets on each radius remained constant in all directions. (2) Pen-movement-distance: the distance to the target was the radius of a circle in which the centre point was the initial position (Figure 2). To examine the relationship between distance and strategy, the distances of 39,131 and 160 pixels $(14.0,47.2$ and $57.6 \mathrm{~mm})$ were determined by a preliminary experiment. (Distances of 39 pixels and 131 pixels were the average values used by ten subjects in a preliminary experiment. When their wrists were in a fixed condition, 39 pixels was the radius of the arc which could be drawn by the subjects; 131 pixels was the radius of the circular arc which was the maximum finger-movement-distance. The outside circle radius of 160 pixels was determined according to the size limitations (height) of the tablet screen. It was also a distance by which the wrist could be moved.). (3) Pen-movement-direction : eight directions were used. They were at $0,45,90,135,180,225,270$ and 315 degrees from the initial position (Figure 2).

The subject had a total of 92 trials for each strategy. These consisted of 20 practice trials and 72 test trials (= 3 target sizes $x 3$ distances $\times 8$ directions).

A break was taken at the end of each strategy trial. Whenever the subject felt tired he/she was allowed to take a rest. Each subject completed 432 test trials $(=6$ strategies $\mathrm{x} 72)$. In each strategy 1512 test trials $(=21$ subjects $\mathrm{x} 72)$ were completed. The order for the six strategies was different for each of the twenty-one subjects.

\section{RESULTS}

An ANOVA (analysis of variance) with repeated measures was performed to determine which strategies and strategy groups were the most efficient. We 
measured these strategies and strategy groups in terms of selection time, error rate, and subjective preference. Moreover, we evaluated the influence of the variables (size, distance, and direction) on the performance differences between these strategies and strategy groups. Error rates were determined by dividing the number of errors by the total number of selection attempts. Selection time was the time required to select the target correctly.

\subsection{Selection Times}

\subsubsection{Comparison of selection times by strategy groups}

Figure 3 shows the selection time for six strategy groups. There was a significant difference between the strategy groups in selection time, $F(5,120)=2.63$, $\mathrm{p}<0.05$. The In-Out strategies were faster (mean $=1.39 \mathrm{~s}$ ) than others. However, there were no significant differences between the On and Off strategies, $F(1,40)=$ $6.01, \mathrm{p}<0.01$, between the $2 \mathrm{D}$ and $3 \mathrm{D}$ strategies, $\mathrm{F}(1,40)=3.66, \mathrm{p}<0.01$, and between the In and In-Out strategies, $F(1,40)=3.37, p<0.01$.

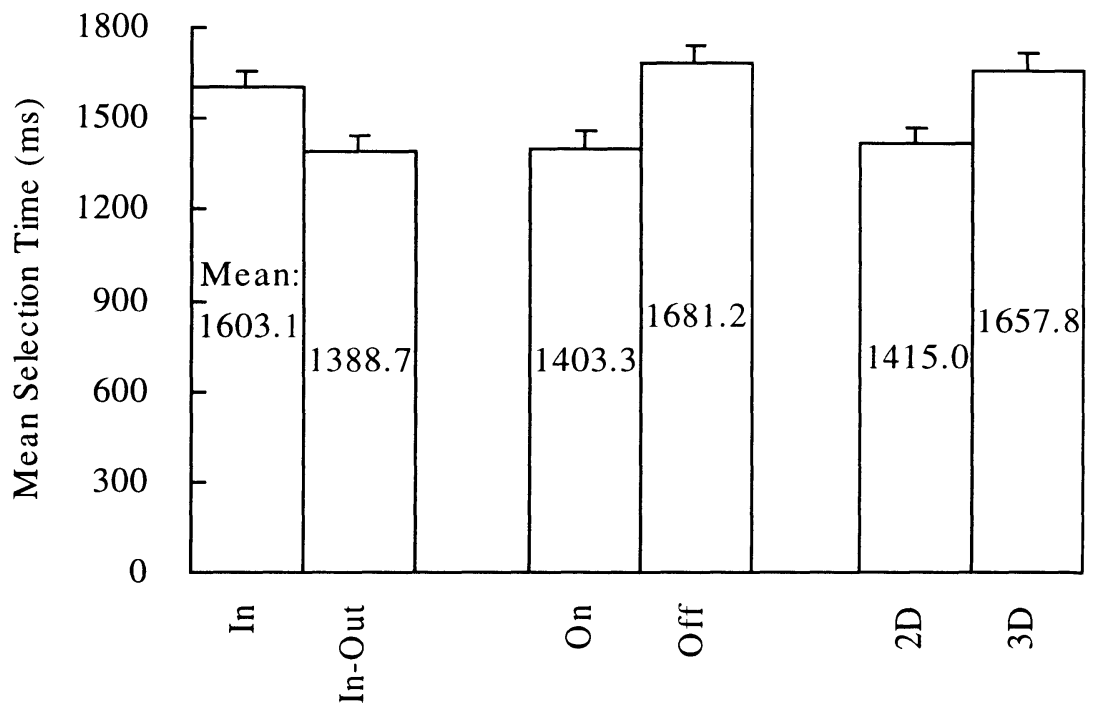

Figure 3 The mean selection time (with standard error bars) for six strategy groups.

\subsubsection{Comparison of selection times by individual strategies}

There was a significant interaction between the six strategies in selection time, $\mathrm{F}(5,120)=10.8, \mathrm{p}<0.0001$. From this we have concluded that the selection time was influenced by the particular strategy, i.e. selection time changed according to 
the strategy being applied. The Land-on2 strategy was the fastest among the six strategies $($ mean $=0.98 \mathrm{~s})$.

Significant interaction was also found between the Land-on2, Take-off 2 and Space2 strategies, $F(2,60)=19.8, p<0.0001$. Analyses were also conducted to determine the significant difference between the six strategies in terms of target size, pen-movement-distance and pen-movement-direction.

- Target size: There were significant differences between the six strategies for each target size, 3,5 , and 9 pixels in selection time, $F(5,120)=9.75,6.85$, and 5.22, $\mathrm{p}<0.001$. This means that significant differences between the six strategies in selection time did not change even when the target size was changed.

- Pen-movement-distance: There were significant differences between the six strategies for each distance, 39,131 , and 160 pixels in selection time, $F(5,120)$ $=7.33,10.3$, and 10.1, $\mathrm{p}<0.0001$.

- Pen-movement-direction: Significant differences in selection time were observed between the six strategies in all directions $0,45,90,135,225,270$ degrees $(p<0.0001$, in case of 180 degrees, $p<0.001)$. 


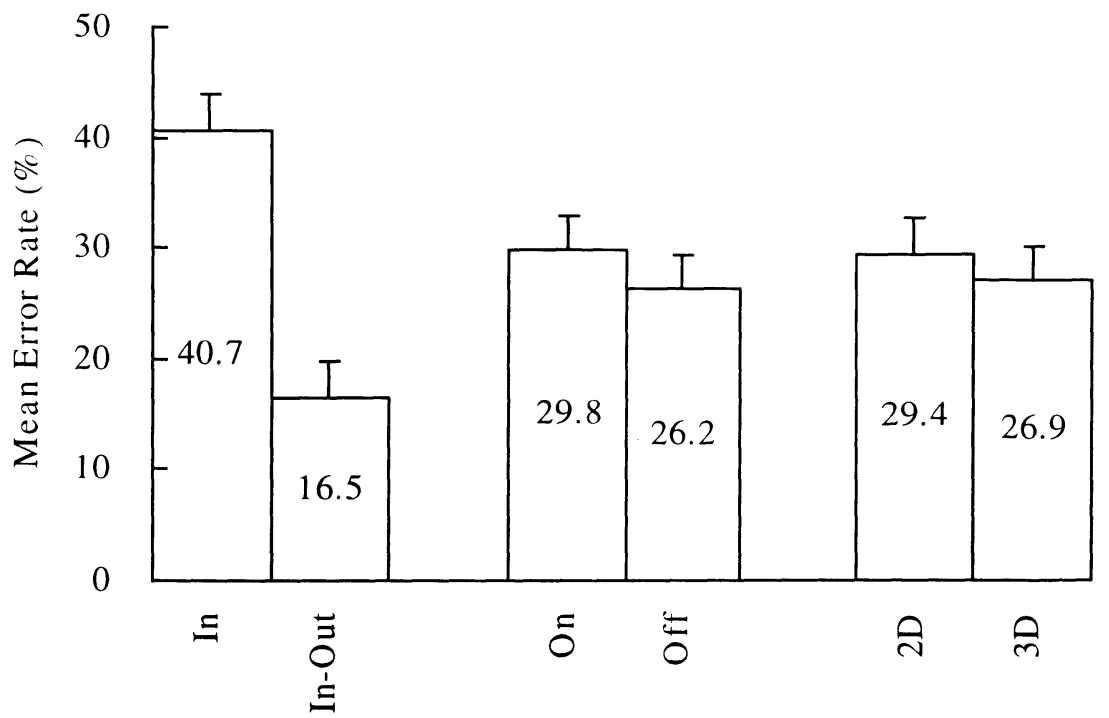

Figure 4 The mean error rate for six strategy groups.

\subsection{Error Rates}

\subsubsection{Comparison of error rates by strategy groups}

Figure 4 shows the error rate for six strategy groups. There was a significant difference among the strategy groups in error rates, $F(5,120)=6.91, p<0.001$. The In-Out strategies had the lowest error rates (16.5\%) and the In strategies had the most error rates $(40.7 \%$ ) among the strategy groups (In, In-Out, On, Off, 2D, and 3D). Moreover, a significant difference was found between the In and In-Out strategies, $\mathrm{F}(1,40)=34.2$, $\mathrm{p}<0.01$, however, significant differences were not seen between the On and Off strategies, $F(1,40)=0.7$, and between the $2 \mathrm{D}$ and $3 \mathrm{D}$ strategies, $\mathrm{F}(1,40)=0.4$.

To investigate the reasons for this, analyses were conducted to determine the significant difference between the In and In-Out strategies in terms of target size, pen-movement-distance and pen-movement-direction.

- Target size: significant differences were found between the In and In-Out strategies for each of the target sizes of 3 and 5 pixels in error rates, $F(1,40)=$ $52.3,18.0, \mathrm{p}<0.01$. On the other hand, there was no significant difference for the target size 9 pixels in error rates, $F(1,40)=1.2, p<0.01$.

- Pen-movement-distance: there were significant differences between the In and In-Out strategies for each distance, 39, 131, and 160 pixels, in error rates, $\mathrm{F}(1,40)=30.2,34.7$, and 33.3, $\mathrm{p}<0.01$. 
- Pen-movement-direction: there were significant differences between the In and In-Out strategies for all eight directions, in error rates, $\mathrm{p}<0.01$.

\subsubsection{Comparison of error rates by individual strategies}

There was a significant difference between the six strategies in error rates, $\mathrm{F}(5,120)=17.8, \mathrm{p}<.0001$. This means that the error rate was influenced by the differences between the strategies. The Land-on2, Take-off 2 and Space2 strategies had lower error rates $(16.6 \%, 17.4 \%$ and $15.5 \%)$ than the other three (Land-on1, Take-off1 and Space1). There was no significant difference between the three (the Land-on2, Take-off2 and Space2 strategies) in error rates, $\mathrm{F}(2,60)=0.08$.

Analyses were also conducted to determine the significant difference between the six strategies in terms of target size, pen-movement-distance and pen-movementdirection.

- Target size: between the six strategies there were significant differences for each of the target sizes of 3 and 5 pixels in error rates, $F(5,120)=24.7,9.99 p$ $<0.0001$. On the other hand, there was no significant difference for the target size 9 pixels on error rates, $F(5,120)=0.65$.

- Pen-movement-distance: there were significant differences between the six strategies for each distance, 39, 131 and 160 pixels, in error rates, $F(5,120)=$ $15.2,16.3$, and 15.5, $\mathrm{p}<0.0001$.

- Pen-movement-direction: there were significant differences between the six strategies for all eight directions in error rates, all at the $\mathrm{p}<0.0001$ level.

\subsection{Subject preferences}

Significant main effects were seen between the six strategies with regard to target size (large targets, $\mathrm{F}(5,30)=14.8, \mathrm{p}<0.0001$, and small targets, $\mathrm{F}(5,30)=$ 58.1, $\mathrm{p}<0.0001$. The Land-on2 and Take-off 2 strategies were rated highly for both large targets and small targets. When selecting a small target, the Land-on2 strategy was the most preferred (mean $=3.08)$.

Figure 5 shows the subject ratings for six strategy groups. Significant differences among the strategy groups were found (large targets, $\mathrm{F}(5,30)=7.01, \mathrm{p}<$ 0.001 , and small targets, $F(5,30)=45.3, p<0.0001$. They were based on the average value of the answers given to the twelve questions by the subjects. The InOut strategy was rated highly for both large targets $($ mean $=4.68)$ and small targets $($ mean $=2.81)$.

From the marks left in Figure 2 by all subjects, we determined that the smallest radius (39 pixels) and the medium radius (131 pixels) were the most popular penmovement-distances. These radii were determined by a preliminary experiment. Though they were radii in which the movements of the hand were few, nevertheless significant differences in the six strategies were observed. There was also a significant difference between the six strategies at the maximum outside radius of 160 pixels. Furthermore, 135, 180, and 225 degrees of pen-movement-direction could be comfortably accommodated. 


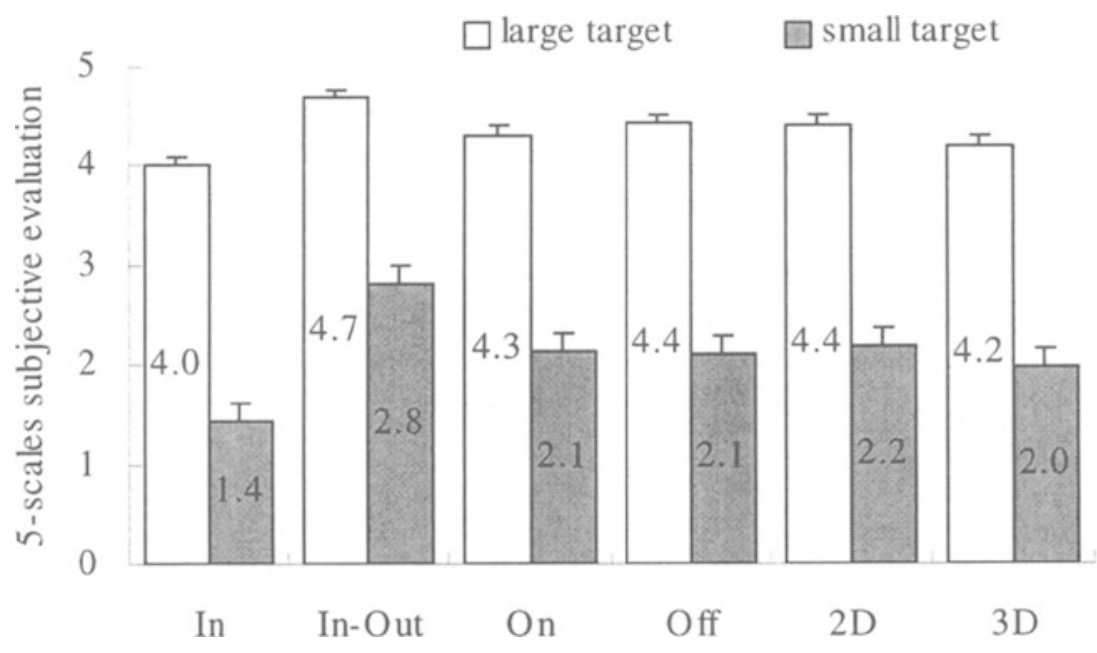

Figure 5 The mean subject ratings for six strategy groups and target sizes $(5=$ highest preference; 1 = lowest preference).

\section{DISCUSSION}

\subsection{The Land-on2 strategy and the In-Out strategy group}

Based on analyses (see 4.) of selection times, error rates and the subjective evaluations, the Land-on2 strategy was the best strategy of the six. This result is the same as the result obtained in another experimental study (Ren and Moriya, 1997a, 1997b). We have verified again that the Land-on2 strategy is the most effective of the six strategies for selecting a small target. Furthermore, the In-Out strategy group was the best among the six strategy groups (In, In-Out, On, Off, 2D and 3D), based on analyses of selection times, error rates, and in particular, the subject preferences (mean $=4.7$ on large targets, 2.8 on small targets in 5 scale rating).

The experimental results show that In-Out strategies (including the Land-on2 strategy) were more efficient than the others. This particularly applies in situations where other targets do not exist near the target, or in situations where they are not too close together, or where other targets do not exist near one side of the target (e.g. the upper part). Sears and Shneiderman (1991) also cite this point with reference to touchscreen situations. For instance, in the Land-on2 strategy, contact with the target may be affected after landing on the screen outside the target area. However, in the Land-on2 strategy, selection is affected on contact with the target area. Since the first target contacted will be selected, prior visual confirmation may be difficult 
to achieve. In this situation, the Take-off 2 strategy can be used because selection does not depend on the point of removal from the screen. Therefore the pen may pass through the target which will not be selected until the pen is removed from any point on the screen.

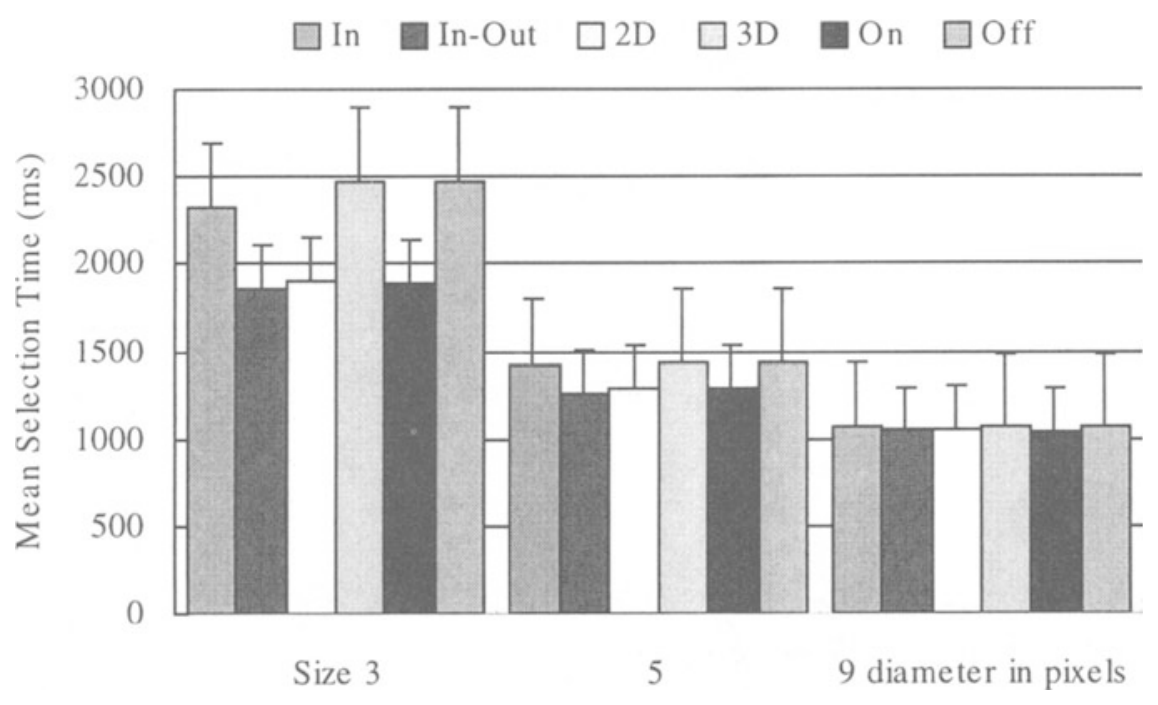

Figure 6 The mean selection time for each strategy group according to target size.

On the other hand, In-Out strategies would not be efficient in the selection of targets in dense displays. Thus, the Land-on 1 and Take-off1 strategies (In strategies) can be used when the screen is a $2 \mathrm{D}$ surface. For instance, the Take-off 1 strategy is the same as the familiar mouse technique. Here the selection is made when the pen contacts the surface of the screen and is moved into the target area after visual confirmation. However, hand/eye coordination is essential when using the Land-on 1 and Take-off 1 strategies. For the Take-off 1 strategy the pen must be within the target (that is, "catching" the target) when the pen is removed from the screen. In the Land-on 1 strategy the pen approaches the screen and target area and it is in the target area only momentarily.

When using an electromagnetic tablet, a target on the screen can be designed as a $3 \mathrm{D}$ target. Thus the Space 1 and Space2 strategies may be used in the same situation. In the Space 1 and Space 2 strategies the pen can affect the target before it makes contact with the screen. 


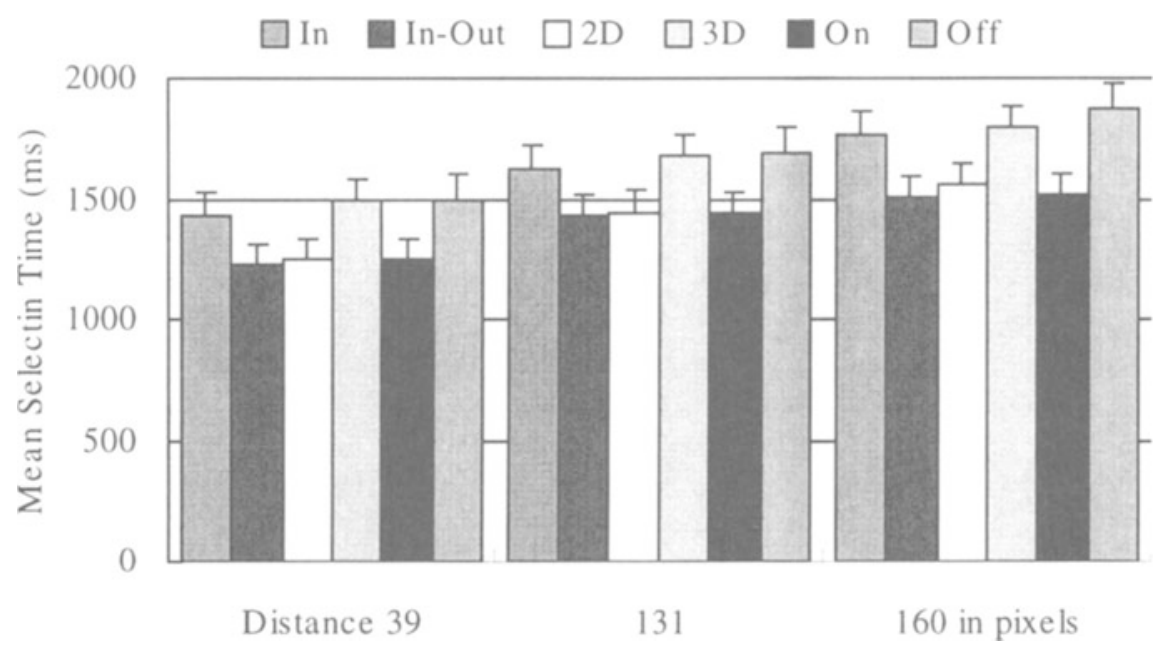

Figure 7 The mean selection time for each strategy group according to distance.

\subsection{Factors influencing the differences between the strategies}

Target size: Regarding target size, there were significant differences between the six strategies in terms of both selection time and error rates for target sizes of 3 pixels and 5 pixels. On the other hand, in the case of the target size of 9 pixels, no significant difference in error rate between the six strategies was observed. The analyses between the In and In-Out strategy groups show the same results. The significant differences between selection strategies were changed by changing the target size. In other words, the error rates were influenced by the selection strategies when the targets were small. These results are important factors in the design of strategies for selecting small targets in pen-based systems.

Pen-movement-distance and pen-movement-direction: It was shown that there were significant differences between the six strategies in both selection time and error rate caused by each of the pen-movement-distances and each of the penmovement-directions. Significant differences in error rate between the In and InOut strategy groups was also observed by each of the pen-movement-distances and each of the pen-movement-directions. This means that significant differences between selection strategies remained in all directions and all distances. Conversely, the differences between the strategies are not changed even when the penmovement-distance or the pen-movement-direction are changed. These results offer some hints for the design of selection strategies. The influence of pen-movementdistance and pen-movement-direction on both error rate and selection time should be considered in pen input strategy design. 


\section{FUTURE RESEARCH}

Fitts' law (Fitts, 1954) states that the time taken to select a target is a function of the size of the target and the distance to the target. There are many variations of this formula. One common form is Movement time $=a+b I D, I D=\log 2$ (Distance / Size +1 ), where, a and b are empirically determined constants, ID is an abbreviation of Index of Difficulty with regard to pointing/selecting. This law has been demonstrated in numerous studies (MacKenzie, 1992). Figure 6 (target size) and Figure 7 (distance) show that selection time gets longer as the target size gets smaller and the distance greater. However, to validate these results additional analyses to plot the selection time against the ID will be conducted. It is not clear that this law, in its original form, applies to all selection methods with all targets. New tasks paradigms presented by Accot and Zhai (1997) may be used to model the Land-on2, Take-off1, and Take-off2 strategies.

The In-Out strategies all allow the user to stray a little from the target and thus the user need not be so accurate. Thus, for small targets, the In-Out strategies reduce selection times and error rates, as confirmed by our results. Future experiments will investigate how far from the target users touch down (in the Landon2 strategy) and how far from the target they take off (in the Take-off2 strategy). This may reveal an effectively larger virtual target.

Comparisons between the Land-on2 strategy and other interaction selecting/pointing techniques should also be conducted to improve the performance of small target acquisition tasks.

It has been reported that differences in target shapes influence the selection time (Sheikh and Hoffmann, 1994). Various target shapes should be used to explore the effects on selection strategies. It is also necessary to investigate the relationships between strategies and target shapes, and to find strategies which are suitable for specific shapes (and vice versa).

\section{CONCLUSIONS}

The paper proposed six strategies and classified the strategies into strategy groups according to their characteristics. It described the experiment in which we compared six strategies for pen-based systems. Experimental results show that the best strategy was the "Land-on2" strategy when the strategies were evaluated individually, and the best strategy group was the "In-Out" strategy group when evaluated in groups. Moreover, the differences between strategies are influenced by variations in target size, however, they are not affected by pen-movement-distance and pen-movement-direction.

We believe that these results will be helpful for designers in identifying and quantifying important factors, and for enhancing user efficiency on pen-based systems. These results may also be useful in future studies to discover new and better strategies. There remains further scope for additional research on the characteristics of pen-based input devices. 


\section{ACKNOWLEDGEMENTS}

We wish to thank the three anonymous reviewers of EHCI 98 for their excellent comments on the draft paper. The TEPCO Research Foundation in Japan has provided the first author with generous financial support for presenting this paper. This support is gratefully appreciated.

\section{REFERENCES}

Accot, J. and Zhai, S. (1997). Beyond Fitts' Law: models for trajectory-based HCI Tasks. Proceedings of the CHI 97 Conference on Human Factors in Computing Systems, pp.295302.

Brewster, S. A., Wright, P. C., Dix, A.J., and Edwards, A.D.N. (1995). The sonic enhancement of graphical buttons. Human-Computer Interaction -INTERACT 95, Lillehammer, pp.43-48.

Fitts, P.M. (1954). The information capacity of the human motor system in controlling amplitude of movement. Journal of Experimental Psychology, Vol.47, No.6, pp.381-391.

Kabbash, P. and Buxton, W. (1995). The "Prince" technique: Fitts' law and selection using area cursors. Proceedings of the CHI95 Conference on Human Factors in Computing Systems, pp.273-279.

MacKenzie, I. S. (1992). Fitts' law as a research and design tool in human-computer interaction. Human-Computer Interaction. 7, pp.91-139.

Potter, R., Weldon, L. and Shneiderman, B. (1988). Improving the accuracy of touch screens: An experimental evaluation of three strategies. Proceedings of the CHI'88 Conference on Human Factors in Computing Systems, pp.27-32.

Ren, X. and Moriya, S. (1995). The concept of various pointing strategies on pen-based computers and their experimental evaluation. Proceedings of the Eleventh Symposium on Human Interface (in Japanese), pp.565-574.

Ren, X. (1996). Pen-based input interfaces for writing and selecting. PhD Thesis (in Japanese), Tokyo Denki University.

Ren, X. and Moriya, S. (1997a). The strategy for selecting a minute target and the minute maximum value on a pen-based computer. Extended Abstract of the CHI97 Conference on Human Factors in Computing Systems, pp.369-370.

Ren, X. and Moriya, S. (1997b). The best among six strategies for selecting a minute target and the determination of the minute maximum size of the targets on a pen-based computer. Human-Computer Interaction -- INTERACT 97, Chapman \& Hall, pp.85-92 .

Ren, X. and Moriya, S. (1998). The Influence of Target Size, Distance and Direction on the Design of Selection Strategies. Proceedings of HCI98, Springer, pp.67-82.

Sears, A. and Shneiderman, B. (1991). High precision touchscreens: design strategies and comparisons with a mouse. International Journal of Man-Machine Studies, 34, pp.593-613.

Sears, A., Plaisant, C. and Shneiderman, B. (1992). A new era for high precision touchscreens. Advances in Human-Computer Interaction, Vol.30, Ablex, Norwood, NJ, pp.1-33.

Sheikh, I. and Hoffmann, E. (1994). Effect of target shape on movement time in a Fitts task. Ergonomics, Vol.37, No.9, pp.1533-1547. 
Soukoreff, W., and Mackenzie, I. S. (1995). Theoretical upper and lower bounds on typing speed using a stylus and soft keyboard. Behaviour \& Information Technology, vol.14, No.6, pp.370-379.

Worden, A., Walker, N., Bharat, K. and Hudson, S. (1997). Making computers easier for older adults to use: area cursors and sticky icons. Proceedings of the CHI97 Conference on Human Factors in Computing Systems, pp.266-271.

Zhai, S., Buxton, W., and Milgram, P. (1994). The "silk cursor": Investigating transparency for 3D target acquisition. Proceedings of the CHI94 Conference on Human Factors in Computing Systems, pp. 459-464.

\section{BIOGRAPHY}

Xiangshi Ren obtained his BS (1991), MS (1993) and Ph.D. (1996) degrees from Tokyo Denki University. He is currently an assistant lecturer in the Department of Information and Communication of Engineering at Tokyo Denki University. His research interests include all aspects of human-computer interaction, in particular, pen-based input interfaces and multimodal interfaces. He is a member of IPSJ, IEICE, and SIGHI of SICE in Japan, and of the British HCI group in UK.

Shinji Moriya received the Ph.D. degree from Tokyo Denki University in 1980. He was visiting associate professor in the State University of New York at Buffalo in 1981 and in the University of Illinois at Urbana-Shampaign in 1982, and visiting professor in the Yunnan Polytechnic University in China in 1994. He is currently professor of Tokyo Denki University. His research interests include pen-based user interactions, voice input user interfaces, and evaluation and modelling of humancomputer interaction. He is a member of IEICE, IPSJ, SICE, Television Society of Japan, ACM and IEEE. 


\section{Discussion}

Philippe Palanque: What about the variable "density of targets?" Usually when you have a small target it means that you have a lot of targets in a small space, thus a high density of possible targets. Have you studied that problem, as it looks like strategy two will lead to a great number of undesired selected targets?

Xiangshi Ren: No, we haven't studied that problem yet. Yes, the density of target displays on small screens is a problem, as you mentioned. Our approach is from the simple to the complex so that we are able to make fundamental comparisons. We feel that research into the complexities relating to multi-target displays with the increased possibility of undesired selection depends on this basic work. We recognize that there will be an infinite number of screen contexts that impact the choice of strategy. Our focus was therefore to discover, describe and measure the essential characteristics and parameters relating to selection strategies as distinct from targets, and target arrangements. We saw this as a necessary precursor to the matters your question raises.

Stefane Chatty: With land-on strategy two, what if I land on a neighbour and then move to the target?

Xiangshi Ren: In the Land-on2 strategy, the target is selected only momentarily at the time the pen makes contact in the target area. If the pen misses the target you can still move the pen to the target to be selected. But if your pen contacts a neighbouring target first an error will occur. By beginning with the simple display of one target, we now have some helpful results which better justify and prepare us for an examination of complex situations.

Ken Fishkin: I worry about relying on selection time as the sole measure of which strategy is best. There are actually two tasks users are performing: acquiring and selecting the target. I might argue that take-off is better, since there is an opportunity to abort. With land-on, there is no abort. Was this measured?

Xiangshi Ren: We measured selection times, error rates, and subjective preferences for six selection strategies in a simple display context. The range of possible solutions for problems relating to variables other than variables in strategy characteristics is potentially infinite. For example, abort functions may or may not be built into a single selection strategy. There are many variables that require examination in their simple form so that solutions to issues of complexity may be resolved on the basis of the results of such research.

Ken Fishkin: Do you have any intuition or experience as to what works best for double-clicking versus single-clicking?

Xiangshi Ren: No, not yet. Our research related only to pen-based devices and functions. The possibility of double tapping with a pen could be researched. 
Stephane Chatty: The pen is already located on the screen, then you move to another target. What if the user's hand is not near the display?

Xiangshi Ren: We need an initial position in order to be able to time selection attempts. The range of possible starting points is perhaps infinite, e.g. hand on the desk, pen in hand, pen not in hand. Our experiment controlled the environment so that the action was measurable.

Jean Scholtz: You have specific distances, e.g., 39 pixels. Why were exactly these distances chosen?

Xiangshi Ren: We performed a preliminary experiment to determine the distances of 39 and 131 pixels. We have reported the details in the body of this paper (refer to Section 3.4). The largest distance (radius) of 160 pixels was determined according to the size limitations of the screen. It was also a distance by which the wrist could possibly be moved. 